\title{
Energy harvesting technologies for wireless sensors in rotating environments
}

\author{
Fredrik Häggström \\ SKF University Technology Center \\ Luleå University of Technology \\ 97187 Luleå \\ Sweden \\ Email: fredrik.haggstrom@ltu.se
}

\author{
Jonas Gustafsson and Jerker Delsing \\ Division of EISLAB \\ Luleå University of Technology \\ 97187 Luleå \\ Sweden
}

\begin{abstract}
Using sensors to measure parameters of interest in rotating environments and communicating the measurements in real-time over wireless links, requires a reliable power source. In this paper, we have investigated the possibility to generate electric power locally by evaluating six different energy-harvesting technologies. The applicability of the technology is evaluated by several parameters that are important to the functionality in an industrial environment. All technologies are individually presented and evaluated, a concluding table is also summarizing the technologies strengths and weaknesses. To support the technology evaluation on a more theoretical level, simulations has been performed to strengthen our claims. Among the evaluated and simulated technologies, we found that the variable reluctancebased harvesting technology is the strongest candidate for further technology development for the considered use-case.
\end{abstract}

\section{INTRODUCTION}

The number of connected devices and sensors are growing every day; Gartner expects that the number of devices connected to Internet of Things will reach 26 Billion units by 2020 representing an almost 30 -fold increase from 0.9 billion in 2009 [1]. To reach this, and even further utilize the potential for wireless sensor systems and Internet of Things in industrial applications, robustness and availability are necessary core features. To achieve these under long-term deployment with minimal manual configuration and maintenance, a reliable energy supply is of utmost importance.

In this paper we investigate technologies to be used for supplying embedded systems in rotating environments (e.g. bearings, shafts or gears) with electric power. Rotating parts are found in almost all machines and are often crucial for the machine operation. From an industrial automation perspective, reduced downtime and increased availability are two aspects that are highly valued. Unexpected faults and downtime sometimes originates from failing rotating parts, which today are monitored to a limited extent. By continuously measuring parameters of interest on vital rotating parts and communicating the information to operators or other parts of the automation system, we can support the prevention of unplanned stops. To communicate the information in a rotating environment, wireless technologies are in general required to avoid electrical contact slip rings. Without cables and slip rings to supply the embedded systems and sensors

\begin{tabular}{llrr}
\hline$n$ & Task & Duration $t_{n}$ & Power $P_{n}$ \\
\hline 0 & Standby & $98 \mathrm{~ms}$ & $0.1 \mathrm{~mW}$ \\
1 & Sampling \& processing & $1 \mathrm{~ms}$ & $10 \mathrm{~mW}$ \\
2 & Receive and transmit & $1 \mathrm{~ms}$ & $60 \mathrm{~mW}$ \\
\hline
\end{tabular}

TABLE I

POWER CONSUMPTION AND DURATION FOR TASKS.

with power, power has to be generated locally and temporary stored within the rotating part or environment. Since batteries have a limited amount of energy, and are often sensitive to temperature variations and other external conditions, battery based solutions are typically not applicable for long-term industrial deployment. The replacements of discharged batteries is also often a costly procedure, especially if the number of deployed sensors (batteries) is great. There are also negative environmental impacts of using large volumes of batteries.

The continued focus of this paper is to evaluate feasible technologies to be used for energy harvesting purpose in rotating environments located in industrial applications.

To limit the paper scope, we have chosen to omit energy harvesting technologies that are based on the physical principles of; heat flow, heat change rate and vibration, as all of these phenomenas are to be avoided or minimized in our use case. Also light and radio frequency based technologies are omitted since they will mostly not be feasible in this context. The technologies we have focused on in this paper all relies on the motion in our rotating environment.

\section{A. Challenges and conditions}

The power consumption for wireless sensors is application specific and depends on several factors; the amount of energy that is used to sample and process data, communicate and idle.

For this paper we estimate the power requirements according to Table I.

$$
P_{\text {avg }}=\frac{\sum_{i=0}^{n} t_{i} P_{i}}{\sum_{i=0}^{n} t_{i}}
$$

By using equation (11) with the estimated values in Table I gives an average power consumption of $0.8 \mathrm{~mW}$. To accom- 


\begin{tabular}{lrl}
\hline Parameter & Value & Unit \\
\hline Rotation frequency & 10 & $\mathrm{~Hz}$ \\
Maximum diameter of rotating env. & 0.1 & $\mathrm{~m}$ \\
Maximum weight of harvesting dev. & 0.05 & $\mathrm{~kg}$ \\
Minimum power output & 1 & $\mathrm{~mW}$ \\
\hline
\end{tabular}

TABLE II

PARAMETERS THAT THE ENERGY HARVESTING DEVICE MUST FULFILL TO COMPLY WITH OUR DEFINED CONSTRAINTS.

modate for conversion losses in the harvesting circuitry, we have defined the minimum required power output from the harvester to $1 \mathrm{~mW}$.

To be able to evaluate the technologies on common ground we have also specified a number of physical and environmental constraints that all investigated technologies must comply with, see Table II These constraints are set with respect to industrial applications, so that many rotating environments found in industries and factories should fulfill these.

To make the energy harvesting technology feasible for use in harsh industrial environments at a reasonable price, a number of conditions has to be considered in this investigation. We chose to highlight the following four parameters to be of high importance, when evaluating different harvesting technologies applicability.

System complexity Including number of components and the challenges involved in getting the system to work as intended. Often also connected with the system cost.

Robustness The ability to withstand harsh external conditions, like dust, dirt, vibrations and large temperature fluctuations.

Generality A measure of how general the technology is, e.g. can the technology be easily applied on several different applications.

Power output The ability to generate electric power.

These parameters are all considered in the technology investigation in the following sections, and are used as the foundation for the concluding technology applicability ranking in the discussion and conclusion section.

\section{B. Related work}

Harvesting ambient energy to power wireless sensor has been used for years. Where several possible sources have been utilized, such as sunlight, indoor lighting, radio frequencies, thermal gradients and vibration as several researchers have summarized [2], [3], [4], [5]. However, none of these sources will be evaluated in this paper since the focus is to convert continuous motion to electrical energy.

Several researcher groups have presented methods to harvest energy from continuous motion, some with focus on lower rotation frequencies than in Table II] e.g. Lafont et al. [6] show how magnetostriction and piezoelectricity can be combined to harvest energy from slow continuous motion. Others are focusing on higher rotation frequencies, e.g. Raisigel et al.

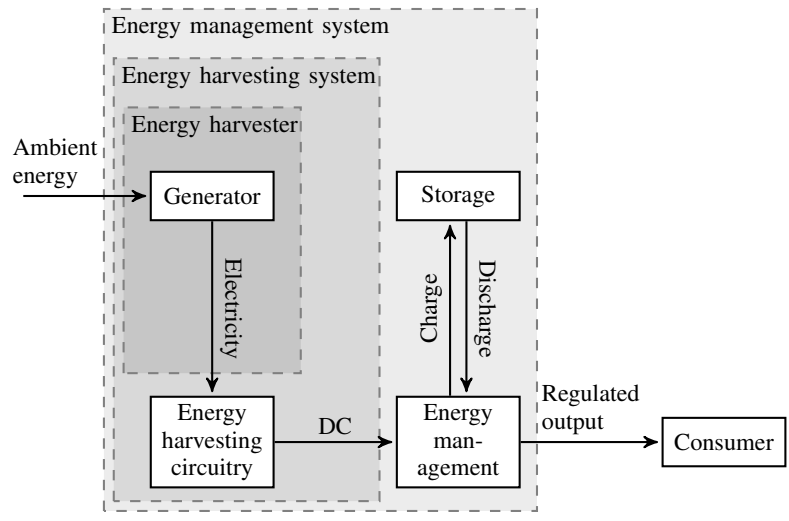

Fig. 1. Definition of an energy management system.

[7] presents a planar micro electromagnetic generator with a power output of $5 \mathrm{~W}$ at a rotation frequency of $6333 \mathrm{~Hz}$. However, based on our findings, there is room for further evaluation and continued research with focus on energy harvesting technologies for operation at rotational frequencies commonly found in conventional industrial equipment. Other important aspects that also need to be considered are that they need to be applicable to several applications, robust and able to withstand long term deployment under harsh conditions.

The main purpose of this paper is to evaluate what appropriate technologies that are available in the literature today and how they could be applied to our use-case to support our continued research on energy harvesting solutions for rotating environments found in industrial machinery.

\section{DESCRIPTION OF ENERGY HARVESTING PRINCIPLES}

Energy harvesters convert non-electrical energy to electricity, as depicted in figure 1. The investigated technologies will act as the generator, harvesting kinetic energy using variable reluctance, electromagnetic induction, wiegand effect, piezoelectricity, magnetostriction or triboelectricity. Where the majority of the investigated harvesting technologies utilizes relative motion between objects to generate electricity. Triboelectric harvesters convert friction, between objects in relative motion to each other, to electricity while variable reluctance, induction- and wiegand harvesters utilize the variation in magnetic field to generate electricity. Piezoelectric- and magnetostriction harvesters rely on induced strain that can generate electrical charges or change a in permeability respectively. The strain in the material can be induced by mechanically stress the material or as Lafont et al. [6] showed by laminating magnetostrictive and piezoelectric materials, which can induce strain by introducing a varying magnetic field.

\section{A. Variable Reluctance}

Variable reluctance sensors are often used to measure rotational speed in rotating systems [8], however the voltage induced in the coil can also be used to supply a wireless sensor with power. In Figure 2 the principle of a variable reluctance sensor is depicted, a permanent magnet generates a magnetic 


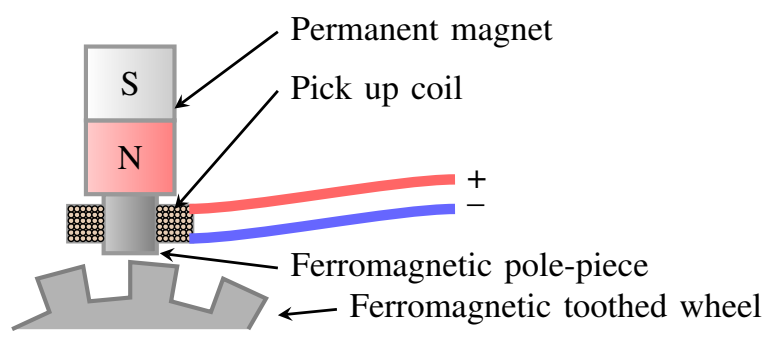

Fig. 2. Cross section of a variable reluctance sensor and a ferromagnetic toothed wheel.

field though a pickup coil with a ferromagnetic core, polepiece. When the ferromagnetic wheel rotates, the magnetic flux path will change, causing a variation in magnetic field through the coil. This will generate a voltage over the coil that can be described by Faraday's law of induction, see equation (2).

$$
V_{e m f}(t)=-N \cdot \frac{d \Phi}{d t}
$$

Where $V_{\text {emf }}$ is the induced voltage in the coil, $N$ is the number of windings in the coil and $\Phi$ is the magnetic flux density trough the loop that the coil forms.

In [8] Parthasarathy et al. shows that it is possible to supply a wireless sensor with enough power using a variable reluctance sensor. If the surrounding material in the rotating system consists of a ferromagnetic material, it could be possible to utilize the rotation of the existing ferromagnetic structure to harvest energy; this opens the possibility to utilize the same harvesting device in many applications.

This makes variable reluctance an interesting technology, which has high robustness and generality and low complexity, which is investigated further.

\section{B. Electromagnetic induction}

Electromagnetic generators are a mature technology as most of the electrical energy in the power grids is generated using this technology. To clarify the difference between electromagnetic induction- and variable reluctance harvesters, electromagnetic induction harvesters are composed of a magnetic source and coil that are in relative motion to each other, while variable reluctance harvesters are composed of a magnetic source and coil that are stationary in relation to each other.

Electromagnetic induction has been utilized in many harvesters to convert rotational motion into electrical energy. In the case where it is not possible to utilize the relative motion between a stationary and rotating system, a pendulum that is joined at the rotation axis of the rotating system can be used. As Toh et al. reports in [9] they use an eccentric weight and DC motor to harvest energy. In cases where the harvester cannot be placed in center of the rotation axis as, the gravitation can be utilized to induce a pendulum motion as the whole harvester is rotated eccentrically, see also [10]. If the rotating and stationary system is in close proximity to each other, the relative motion between the systems can be

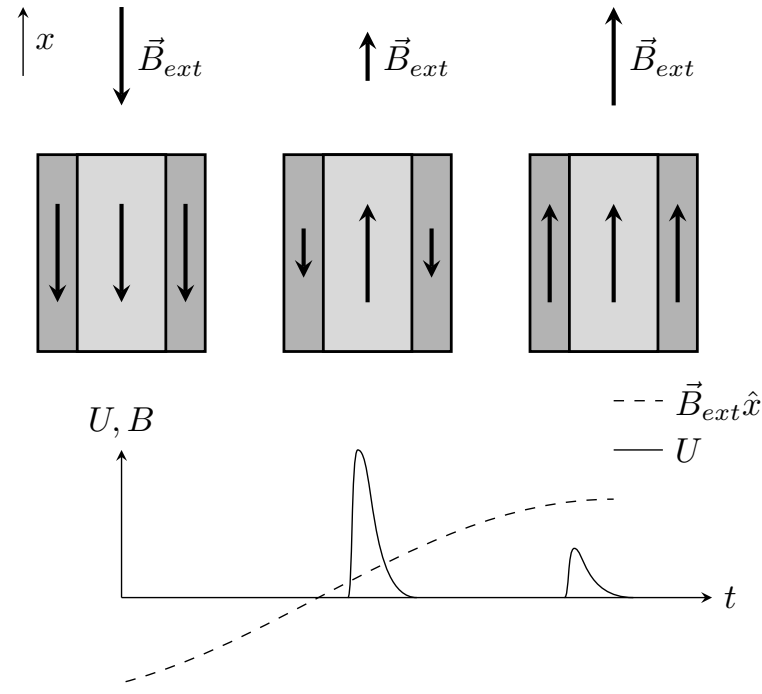

Fig. 3. Crossection of a wiegand wire switching polarity when a varying external magnetic field is introduced, which generates two voltage peaks in the coil that is wound around the wiegand wire.

used to induce a varying magnetic field by adding a magnetic source in one of the systems and a coil in the other.

Electromagnetic harvesters that receive a varying magnetic field from relative movements from permanent magnets can generate electrical energy without active circuitry and startup energy. This gives electromagnetic induction harvesters average complexity and generality and high robustness.

\section{Wiegand effect}

Wiegand wires are composed of a special annealed alloy that is cold-worked by twisting it back and forth to induce strain in the outer shell of the wire, while the center has less residual strain. This creates an outer shell of the wire that is hardened and has a higher magnetic hysteresis than the center that is still "soft" with a lower magnetic hysteresis [11]. By having a wire with a soft center and a hardened outer shell, two dominating magnetic domains are created.

As an external varying magnetic field $\vec{B}_{\text {ext }}$ is introduced the inner and outer magnetic domains will change polarity in steps as shown in figure 3 . First, the core will change polarity in the direction of the external magnetic field and generate a voltage peak in the coil. Secondly, the outer core will change its polarity in the direction of the external magnetic field. This will also generate a voltage peak, although not as high as when the core changed direction.

The magnetic polarity switch event can be picked up by a coil that will generate a fixed amount of energy for each switching event, which depends on the alloy and geometry of the wiegand wire [12]. This effect has a low dependence of the external magnetic field frequency, thus making this it possible to use with slowly rotating systems as Ongaro et al. reports [13].

The energy generated from each switching event is too low to generate $1 \mathrm{~mW}$ with the current constraints from Table II] 


\section{Piezoelectric}

Piezoelectric materials are commonly used to generate electricity from vibrations due to the simple conversion from mechanical- to electrical domain. By letting a mechanical resonator circuit put cyclic stress on the piezoelectric material, the piezoelectric material will generate a charge as the material is deformed [14]. The earth's gravity in combination with a rotating motion of the system can be used to induce an varying force to excite the mechanical resonator circuit as $\mathrm{Gu}$ et al. reports [15]. In the paper, it is shown that the centripetal force can be used to self-tune the mechanical resonator to make the resonator operate at a broader range of frequencies.

Another way piezoelectric materials can be used is to attach it to parts of the mechanical system that deforms as the system rotates, it could generate enough charge to power a wireless sensor.

Piezoelectric harvesters mainly rely on resonance at a specific frequency. This limits the applicability of the harvester and lowers generality, and findings within the constraints of Table III shows that the power output would be lower than $1 \mathrm{~mW}$. However, a harvester that relies on deformations of the system might be of interest.

\section{E. Magnetostrictive}

If magnetostricitve materials are subjected to a varying magnetic field, the dimensions of the material will change. By laminating highly magnetostrictive and piezoelectric materials, strain can be induced in the piezoelectric material by a timevarying magnetic field thus generateing a charge from a time-varying magnetic field [16]. Since piezoelectric materials have a high internal resistance, the charge generated in the piezoelectric material from the induced strain will dissipate slowly.

Hence this technology can be used for slow time-varying magnetic fields as Lafont et al. reports [6]. However using this technology within the constrains in Table II and with the high magnetic field that is necessary for the magnetostrictive material to induce strain on the piezoelectric material, a lot of eddy current would be generated thus making the energy conversion inefficient.

\section{F. Triboelectric}

If the stationary and rotating system are in close proximity to each other, a triboelectric harvester could be used. Triboelectric harvesters convert friction between objects into electrical charges. The triboelectric effect is often an undesirable effect in a system; charges generated through the effect can destroy electronics and create discharges.

Zhu et al. have shown a triboelectric harvester that converts rotary motion into electricity [17]. They claim to harvest $1.5 \mathrm{~W}$ at a rotation frequency of $50 \mathrm{~Hz}$. They also claim that the power output is linearly dependent on the rotation frequency, hence the power output at $10 \mathrm{~Hz}$ would be $300 \mathrm{~mW}$.

This technology might be of interest if it can be shown that the friction does not degrade the material over time. However, this technology increases complexity due to tolerances and cleanliness.

\section{G. Technology Comparison}

The individual components used in variable reluctance-, electromagnetic- and wiegand harvester are considered robust by us since permanent magnets and coils can withstand temperatures above $100^{\circ} \mathrm{C}$ without degrading significantly [18] and are not sensitive to dust and dirt. The wiegand effect can also be observed up to $125^{\circ} \mathrm{C}$ in commercial sensors [19] and neither the ferromagnetic material, permanent magnets or coils are sensitive to vibrations or wear due to the non-contact harvesting principle.

The electromagnetic and wiegand harvesters have to introduce components in both the rotating and stationary system that adds complexity, increases cost and reduces the generality. The variable reluctance harvester only requires introducing components in one of the systems (depending on the specific application) if it is deployed in a close proximity to a relative moving ferromagnetic environment.

The voltage output from variable reluctance- and electromagnetic harvesters dependings on the rotation frequency. If the voltage generated from the harvester is below the minimum voltage required for the consumer circuitry plus the forward voltage drop from a rectifying bridge, the consumer circuitry will not be able to operate with a passive solution. Hence active circuits can be utilized to boost the voltage as several researchers reports [20], [21] which enables the harvester to operate at lower rotation frequencies. However, the active harvesting circuitry requires a start-up voltage, which means either that the wireless sensor is dependent on long-term energy storage, or that the harvester might experience a higher rotation frequency in periods of time that generates a higher voltage, which allows the active harvesting circuitry to startup. After the active harvesting circuitry is operational, the rotation frequency can decrease and the consumer circuitry can still be operational.

Piezoelectric harvesters often utilizes lead zirconate titanate (PTZ) films [2], [3], [4], [5] which have a higher ageing rate than permanent magnets [18], [22]. This will decrease the power output of the harvester over time, which lowers the robustness of such a system. A passive rectifying bridge can convert the electricity to DC due to the high voltages generated in the piezoelectric material, however active solutions can be used to increase the power output as several papers suggest [23], [24], [25]. Piezoelectric harvesters must be tuned to each specific application and cannot work for a broad range of frequencies, which increases complexity and decreases generality.

A magnetostrictive harvester that relies on inducing strain in a piezoelectric material requires an intense magnetic field that varies over time. The induced eddy currents in the magnetostrictive material will increase with the rotation frequency, as described in [26], this will generate heat in the magnetostrictive material and make the conversion inefficient. 
However, this technology is well suited for applications with lower rotation frequencies than we are addressing in this paper. Similar harvesting circuits as for piezoelectric harvesters can be used. This technology also requires components in both the rotating and stationary system, which increases complexity and decreases generality.

Triboelectric harvesters require parts in both the rotating and stationary system, which increase the complexity. Zhu et al. showed a test running for $\sim 1.7 \times 10^{5}$ revolutions without any decay in power output. However this corresponds to only $\sim 4.5$ hours of operation at a rotation frequency of $10 \mathrm{~Hz}$. It would be interesting to see a long-term test of such a harvester.

Since no long-term performance test of a triboelectric harvester could be found, it is assumed that the friction between the parts will cause ware and shorten the lifetime of the harvester, thus decreasing the robustness.

All the finding have been graded and summarized in Table III Robustness, complexity and generality is described in section I-A Robustness is graded between 1 to 5 , where 1 means poor and 5 means good robustness. Complexity is graded between 1 to 5 , where 1 means low and 5 means high complexity. Generality is graded between 1 to 5 , where 1 means poor and 5 means good generality.

\section{H. Comparison Outcome}

From Table [II it can be seen that a variable reluctance harvester that is operating within the constraints in Table II has lowest complexity, high robustness and highest generality. A variable reluctance harvester would also provide enough energy to supply the assumed system in Table I This implies that more investigation within this technology was needed to verify the credentials of the investigation. Hence, a simulation was conducted on how a variable reluctance harvester would perform under the defined constraints.

\section{Simulation}

In order to optimize spatial dimensions of the harvester and confirm that variable reluctance can generate enough energy yielding the defined constraints, a simulation in COMSOL $4.3 \mathrm{~b}$ was conducted. A ferromagnetic toothed wheel with a relative permeability of 100 , an outer diameter of $10 \mathrm{~cm}$ and 50 teeth was used as the rotating part in the simulation, with a rotation frequency of $10 \mathrm{~Hz}$.

The variable reluctance harvester was composed of a ferromagnetic pole-piece with 4000 in relative permeability formed as a solid cylinder with a diameter of $2 \mathrm{~mm}$ and a length of $4 \mathrm{~mm}$. Around the pole-piece a copper wire with a diameter of $0.03 \mathrm{~mm}$ is wound with 5800 revolutions. The resistance of the wire in the coil is calculated to $500 \Omega$, due to the length and area of the wire, which also is the internal resistance and dominating part of the harvesters impedance.

On top of the pole-piece a cylindrical neodymium magnet with a diameter of $5 \mathrm{~mm}$ and length of $4 \mathrm{~mm}$ was placed, with a remanent flux density of $1.2 \mathrm{~T}$ in the direction towards the pole-piece. Two simulations were conducted, one with the harvester mounted so the pole-piece had an airgap of $1 \mathrm{~mm}$ and

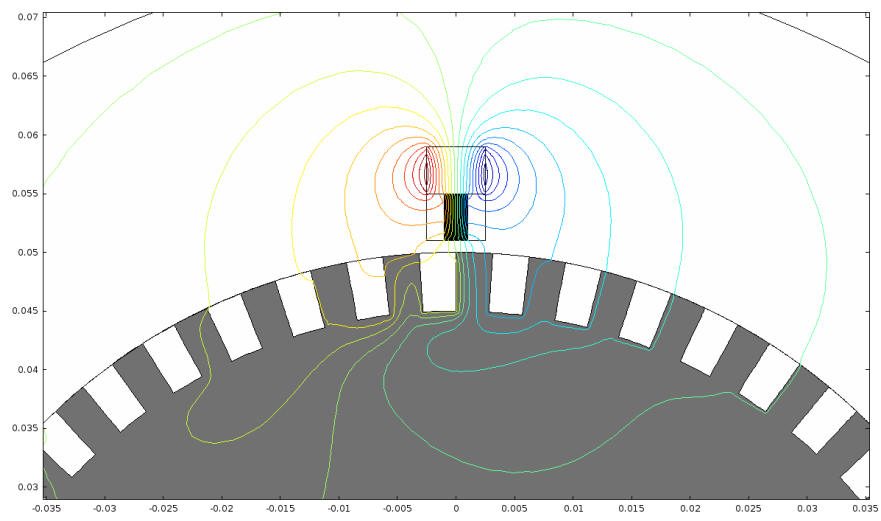

Fig. 4. Overview of the simulation model. Induced voltage

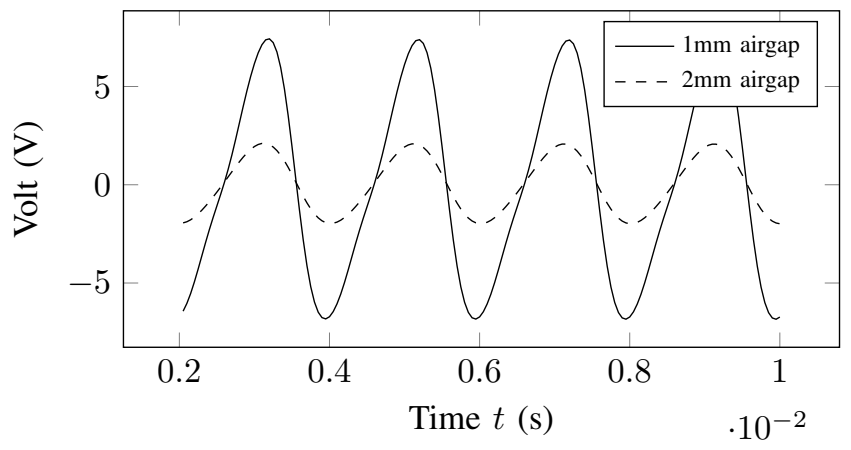

Fig. 5. The figure shows the simulated induced voltage, generated by the variable reluctance simulation model described in the text.

another with the pole-piece $2 \mathrm{~mm}$ from the rotating toothed wheel, see Figure 4 for a screenshot of the simulation model.

The simulation results show that this energy harvesting setup can produce $\sim 4.9 \mathrm{~V}_{\text {rms }}$ at $1 \mathrm{~mm}$ airgap and $\sim 1.4 \mathrm{~V}_{\text {rms }}$ with a $2 \mathrm{~mm}$ airgap, see Figure 5 Which yields an extractable power of $12 \mathrm{~mW}$ and $1 \mathrm{~mW}$ respectively, when connected to a load matching the internal impedance of the harvester which in this simulation is $500 \Omega$. This result is similar to what Parthasarathy et al. showed in their experimental setup, found in [8].

\section{DISCUSSION AND CONCLUSION}

As embedded systems and wireless sensors merge with mechanical parts such as bearings and other rotating environments, the need for energy harvesting technologies increases. In this paper, we have evaluated six different energy-harvesting principles that are possible to use in rotating environments.

Among the technologies included in this technology evaluation, we found that the variable reluctance-based energy harvesting technology is the most promising for the addressed application; a rotating object that complies to Table III

We found that there are many possible technologies to be applied for energy harvesting purpose in rotating environments. However, many technologies did not meet our 
TABLE III

SUMMARY OF ENERGY HARVESTING TECHNOLOGIES.

\begin{tabular}{|c|c|c|c|c|}
\hline Technology & Power $[\mathrm{mW}]$ & Robustness [1-5] & Complexity [1-5] & Generality [1-5] \\
\hline Variable reluctance & $2 *[8$ & 5 & 1 & 5 \\
\hline Electromagnetic & $5.6^{*}[9]$ & 5 & 3 & 3 \\
\hline Wiegand & $7.6 \times 10^{-3} * 13$ & 5 & 3 & 3 \\
\hline Piezoelectric & $0.18^{*}$ & 3 & 3 & 2 \\
\hline Magnetostrictive & $3.2 * \sqrt{6}$ & 3 & 4 & 1 \\
\hline Triboelectric & $300[17$ & 1 & 3 & 3 \\
\hline
\end{tabular}

* inter-/extrapolated from reference result.

requirements, as they did not produce enough power with our defined conditions or had poor robustness, generality and high complexity in our defined environment. However, these might still be interesting for further investigation in other application, e.g. very slow/high rotation frequencies, extreme temperature conditions, etc.

\section{ACKNOWLEDGEMENT}

The authors would like to thank SKF AB and the Arrowhead project for their contributions and valuable discussions.

\section{REFERENCES}

[1] "Forecast: The internet of things, worldwide, 2013."

[2] J. Matiko, N. Grabham, S. Beeby, and M. Tudor, "Review of the application of energy harvesting in buildings," Measurement Science and Technology, vol. 25, no. 1, p. 012002, 2014.

[3] S. P. Beeby, M. J. Tudor, and N. White, "Energy harvesting vibration sources for microsystems applications," Measurement science and technology, vol. 17, no. 12, p. R175, 2006.

[4] A. Harb, "Energy harvesting: State-of-the-art," Renewable Energy, vol. 36, no. 10, pp. 2641-2654, 2011.

[5] A. Khaligh, P. Zeng, and C. Zheng, "Kinetic energy harvesting using piezoelectric and electromagnetic technologiesi $i_{i}^{1 / 2}$ state of the art," Industrial Electronics, IEEE Transactions on, vol. 57, no. 3, pp. 850-860, 2010.

[6] T. Lafont, L. Gimeno, J. Delamare, G. Lebedev, D. Zakharov, B. Viala, O. Cugat, N. Galopin, L. Garbuio, and O. Geoffroy, "Magnetostrictivepiezoelectric composite structures for energy harvesting," Journal of Micromechanics and Microengineering, vol. 22, no. 9, p. 094009, 2012.

[7] H. Raisigel, O. Cugat, and J. Delamare, "Permanent magnet planar micro-generators," Sensors and Actuators A: Physical, vol. 130, pp. 438444, 2006.

[8] D. Parthasarathy, P. Enoksson, and R. Johansson, "Prototype energy harvesting wheel speed sensor for anti-lock braking," in Robotic and Sensors Environments (ROSE), 2012 IEEE International Symposium on. IEEE, 2012, pp. 115-120.

[9] T. T. Toh, P. D. Mitcheson, A. S. Holmes, and E. M. Yeatman, "A continuously rotating energy harvester with maximum power point tracking," Journal of Micromechanics and Microengineering, vol. 18, no. 10, p. 104008, 2008.

[10] Y.-J. Wang, C.-D. Chen, and C.-K. Sung, "Design of a frequencyadjusting device for harvesting energy from a rotating wheel," Sensors and Actuators A: Physical, vol. 159, no. 2, pp. 196-203, 2010.

[11] "Bistable magnetic device," Jun. 25 1974, uS Patent 3,820,090.

[12] P. E. Wigen, "Wiegand wire: New material for magnetic-based devices," Electronics, vol. 48, pp. 100-105, 1975.

[13] F. Ongaro, S. Saggini, and L. Corradini, "Low-power energy harvester for wiegand transducers," in Applied Power Electronics Conference and Exposition (APEC), 2013 Twenty-Eighth Annual IEEE, 2013, pp. 453459.

[14] T. J. Kazmierski and S. Beeby, Energy Harvesting Systems. Springer, 2011.
[15] L. Gu and C. Livermore, "Passive self-tuning energy harvester for extracting energy from rotational motion," Applied Physics Letters, vol. 97, no. 8, pp. 081904-081904, 2010.

[16] O. Geoffroy, D. O'Brien, O. Cugat, and J. Delamare, "Practical and theoretical investigations of a rotating coilless actuator using the inverse magnetostrictive effect," Magnetics, IEEE Transactions on, vol. 46, no. 2, pp. 606-609, 2010.

[17] G. Zhu, J. Chen, T. Zhang, Q. Jing, and Z. L. Wang, "Radial-arrayed rotary electrification for high performance triboelectric generator," Nature communications, vol. 5, 2014.

[18] Alliance LLC, "Magnet guide \& tutorial." [Online]. Available: http://www.allianceorg.com/pdfs/Magnet_Tutorial_v85_1.pdf

[19] Nanjing AH Electronic Sci \& Tech Co Ltd., "Manufacturerï i1/2s Specification of WG Series Wiegand Sensors and Wiegand Modules." [Online]. Available: http://www.elecrow.com/download/K5004477604. pdf

[20] E. Arroyo, A. Badel, and F. Formosa, "Energy harvesting from ambient vibrations: Electromagnetic device and synchronous extraction circuit," Journal of Intelligent Material Systems and Structures, 2013.

[21] P. D. Mitcheson, T. C. Green, and E. M. Yeatman, "Power processing circuits for electromagnetic, electrostatic and piezoelectric inertial energy scavengers," Microsystem Technologies, vol. 13, no. 11-12, pp. 1629-1635, 2007.

[22] W.-P. Tai and S.-H. Kim, "Relationship between cyclic loading and degradation of piezoelectric properties in $\mathrm{pb}(\mathrm{zr}, \mathrm{ti}) \mathrm{o}_{i} \mathrm{sub}_{i}, 3 \mathrm{i} / \mathrm{sub}_{i}$ ceramics," Materials Science and Engineering: B, vol. 38, no. 1, pp. 182-185, 1996

[23] E. Lefeuvre, A. Badel, C. Richard, and D. Guyomar, "Piezoelectric energy harvesting device optimization by synchronous electric charge extraction," Journal of Intelligent Material Systems and Structures, vol. 16, no. 10, pp. 865-876, 2005.

[24] D. Guyomar, A. Badel, E. Lefeuvre, and C. Richard, "Toward energy harvesting using active materials and conversion improvement by nonlinear processing," Ultrasonics, Ferroelectrics and Frequency Control, IEEE Transactions on, vol. 52, no. 4, pp. 584-595, 2005.

[25] Y. Wu, A. Badel, F. Formosa, W. Liu, and A. Agbossou, "Selfpowered optimized synchronous electric charge extraction circuit for piezoelectric energy harvesting," Journal of Intelligent Material Systems and Structures, p. 1045389X13517315, 2014.

[26] H. Savage et al., "Theory and application of highly magnetoelastic metglas 2605sc," Journal of Applied Physics, vol. 53, no. 11, pp. 8092$8097,1982$. 